\title{
Genetic Profiles of Ferroptosis in Malignant Brain Tumors and Off-Target Effects of Ferroptosis Induction
}

\author{
Marc Dahlmanns $^{1 *}$, Eduard Yakubov ${ }^{2}$ and Jana Katharina Dahlmanns ${ }^{3+}$ \\ 1 Institute for Physiology and Pathophysiology, Friedrich-Alexander-University Erlangen-Nuremberg, Erlangen, Germany, \\ ${ }^{2}$ Department of Neurosurgery, Paracelsus Medical University, Nuremberg, Germany, ${ }^{3}$ Independent Researcher, \\ Erlangen, Germany
}

OPEN ACCESS

Edited by: Jürgen Schlegel, Technical University of Munich,

Germany

Reviewed by:

Michael Wayne Epperly, University of Pittsburgh, United States Chunming Cheng,

The Ohio State University,

United States

*Correspondence:

Marc Dahlmanns

marc.dahlmanns@fau.de

+ORCID:

Jana Katharina Dahlmanns orcid.org/0000-0002-5791-4888

Specialty section:

This article was submitted to Neuro-Oncology and

Neurosurgical Oncology,

a section of the journal

Frontiers in Oncology

Received: 25 September 2021 Accepted: 15 November 2021 Published: 01 December 2021

Citation:

Dahlmanns $M$, Yakubov E and Dahlmanns JK (2021) Genetic Profiles of Ferroptosis in Malignant Brain Tumors and Off-Target Effects of Ferroptosis Induction.

Front. Oncol. 11:783067. doi: 10.3389/fonc.2021.783067
Glioblastoma represents the most devastating form of human brain cancer, associated with a very poor survival rate of patients. Unfortunately, treatment options are currently limited and the gold standard pharmacological treatment with the chemotherapeutic drug temozolomide only slightly increases the survival rate. Experimental studies have shown that the efficiency of temozolomide can be improved by inducing ferroptosis - a recently discovered form of cell death, which is different from apoptosis, necrosis, or necroptosis and, which is characterized by lipid peroxidation and reactive oxygen species accumulation. Ferroptosis can also be activated to improve treatment of malignant stages of neuroblastoma, meningioma, and glioma. Due to their role in cancer treatment, ferroptosis-gene signatures have recently been evaluated for their ability to predict survival of patients. Despite positive effects during chemotherapy, the drugs used to induce ferroptosis - such as erastin and sorafenib - as well as genetic manipulation of key players in ferroptosis - such as the cystine-glutamate exchanger $\mathrm{xCT}$ and the glutathione peroxidase GPx4 - also impact neuronal function and cognitive capabilities. In this review, we give an update on ferroptosis in different brain tumors and summarize the impact of ferroptosis on healthy tissues.

Keywords: ferroptosis, neuroblastoma, glioblastoma, erastin, neuron, xCT, brain tumor therapy, off-target effects

\section{INTRODUCTION}

Ferroptosis is as an iron-dependent form of cell death, which is different from previously known forms of cell death such as apoptosis, necrosis, or necroptosis. It is characterized by the accumulation of reactive oxygen species (ROS) and lipid peroxidation (1-3). After finding that activating ferroptosis in cancer cells of mice improved the effectiveness of temozolomide treatment - a first-line chemotherapeutic drug against glioblastoma (glioma WHO grade IV) $(4,5)$ - further investigations revealed the important role of ferroptosis also in human cancer patients.

Glioma is a type of primary brain tumor that is generated from glial cells in the central nervous system. These gliomas are classified by the WHO into low-grade glioma (WHO grade II) and highgrade glioma (WHO grade III/IV), where higher grading is associated with poorer prognosis (6). 
Ferroptosis represents an option to improve treatment for patients suffering especially from these more malignant tumors, including glioblastomas, because these are difficult to cure by radiation, resection, or pharmacological treatment alone. Especially because pharmacological treatment is affected by drug resistances (7).

Since the discovery of ferroptosis in 2012 (1) several key molecules have been identified, which are either directly integrated into the ferroptosis process or act as inducers. Current data about key players in ferroptosis and their role in glioma have been reviewed elsewhere $(8,9)$. The recently launched database ferrDB provides an overview of these regulators and markers in ferroptosis (10).

This review provides an overview of ferroptosis in the therapy of various brain tumors with a focus on ferroptosis gene signatures, which have a strong translational value in predicting patients' prognosis, and of the effects of ferroptosis induction in non-cancerous tissue that is also affected during treatment (Figure 1).

\section{PROMISING FINDINGS ON FERROPTOSIS INDUCTION IN NEUROBLASTOMA AND MENINGIOMA}

Expanding on the treatment boosting effects of ferroptosis induction in glioma, there are also promising findings in other types of cancer. Neuroblastoma is a highly relevant pediatric cancer in younger children (11), with limited treatment options and therapy resistance if occurring in its high-risk form (12). Induction of ferroptosis to limit tumor growth has been emerging as a striking new concept to treat neuroblastoma.

Ferroptosis can be induced by several small molecules [as reviewed elsewhere (8)] or by inhibition of the glutathione peroxidase GPx4 (13) and glutamate/cystine antiporter system $\mathrm{x}_{\mathrm{c}}^{-}$(SLC7A11; also referred to as $\mathrm{xCT}$ ) through the drugs erastin (1), sulfasalazine (14), or sorafenib (15), amongst others.

Recently, treatment with the steroidal lactone withaferin A was found to induce the nuclear factor erythroid 2-related factor 2 (Nrf2) pathway and to inactivate the GPx4 pathway, a duality making this strategy highly effective in treating both neuroblastoma cells and transplanted xenografts in mice (16). In this study the substance was targeted to the tumor site with nanoparticles, which reduces side-effects (17).

Chemosensitization to erastin-induced ferroptosis was also accomplished after knockdown of the iron exporter ferroportin in neuroblastoma SH-SY5Y cells (18).

In about $25 \%$ of neuroblastoma cases MYCN is amplified (19). In patient-derived xenografts of these cases, the xCT-driven antioxidant response after sulfasalazine application is increased compared to controls, which leads to an increase in ferroptosis and subsequently limited tumor growth (19). Further studies revealed that the transferrin receptor 1 was upregulated in response to such MYCN amplification, leading to increased GPx4 sensitivity and rendering neuroblastoma cells vulnerable to ferroptosis induction (20). In addition to this genetically mediated sensitization, the inhibition of PKC $\alpha$ stimulated ferroptosis and sensitized neuroblastoma stem cells to etoposide, which is particularly relevant given the central role of stem cells in conferring resistance to therapy (21). Neuroblastoma cell lines also express a very low level of ferritin heavy chain 1, whose reduction leads to a rise in ROS and a higher sensitivity to ferroptosis (22). In meningioma cell lines derived from patients covering WHO Grades I-III, the vulnerability to erastin-induced ferroptosis was increased both by a loss of neurofibromin and by a low level of E-cadherin. The expression of these proteins is driven by the myocyte enhancer factor $2 \mathrm{C}$, making it a promising factor to manipulate during meningioma treatment $(23,24)$.

In summary, treatment of cancers such as neuroblastoma and meningioma in their advanced stages may be improved by exploiting the role of ferroptosis.

\section{FERROPTOSIS-GENE SIGNATURES IN GLIOMA}

Gliomas represent a major form of brain cancer, divided into WHO grades I to IV with glioblastoma being the most devastating form of human brain cancer (6) because it is associated with a low survival, therapy resistance and limited treatment options (25). To overcome these obstacles, genetic studies based on large patient databases have examined the link between gene expression in glioma and overall survival in riskstratified patient cohorts. In these studies, ferroptosis- and glioma-related genes of interest were identified by screening RNA sequencing data and associated clinical data. These genesignatures constitute a risk-model, predicting the overall survival of the patients. To avoid overfitting, the models were each constructed in one database, e. g. Chinese Glioma Gene Atlas (CGGA), and validated using other databases, e. g. Repository for Molecular Brain in Neoplasia Data (REMBRANDT) or The Cancer Genome Atlas (TCGA) (26-32). The risk-models are shown and described in Table 1. The risk models that are based on the ferroptosis-related genes stratified glioma patients into a low-risk and high-risk cohort.

In high-risk cohorts, the median survival probabilities indicated by Kaplan-Meier curves were significantly decreased. The risk-score was often correlated with clinicopathological features such as the WHO grade or the O-6-Methylguanine-DNA Methyltransferase (MGMT) promotor methylation status, proving the suitability of ferroptosis-related gene expression pattern for patient outcome prediction. Interestingly, functional annotation of the ferroptosisrelated genes in the risk-models revealed that often the immune system is involved: Investigation of RNA sequencing data from glioblastoma (TCGA) revealed that the expression levels of ferroptosis suppressors such as CD44, HSPB1 and SLC40A1 correlated with the degree of immunosuppression and were related to survival of patients (34). The expression of these suppressors could also be induced by acetaminophen (34). 


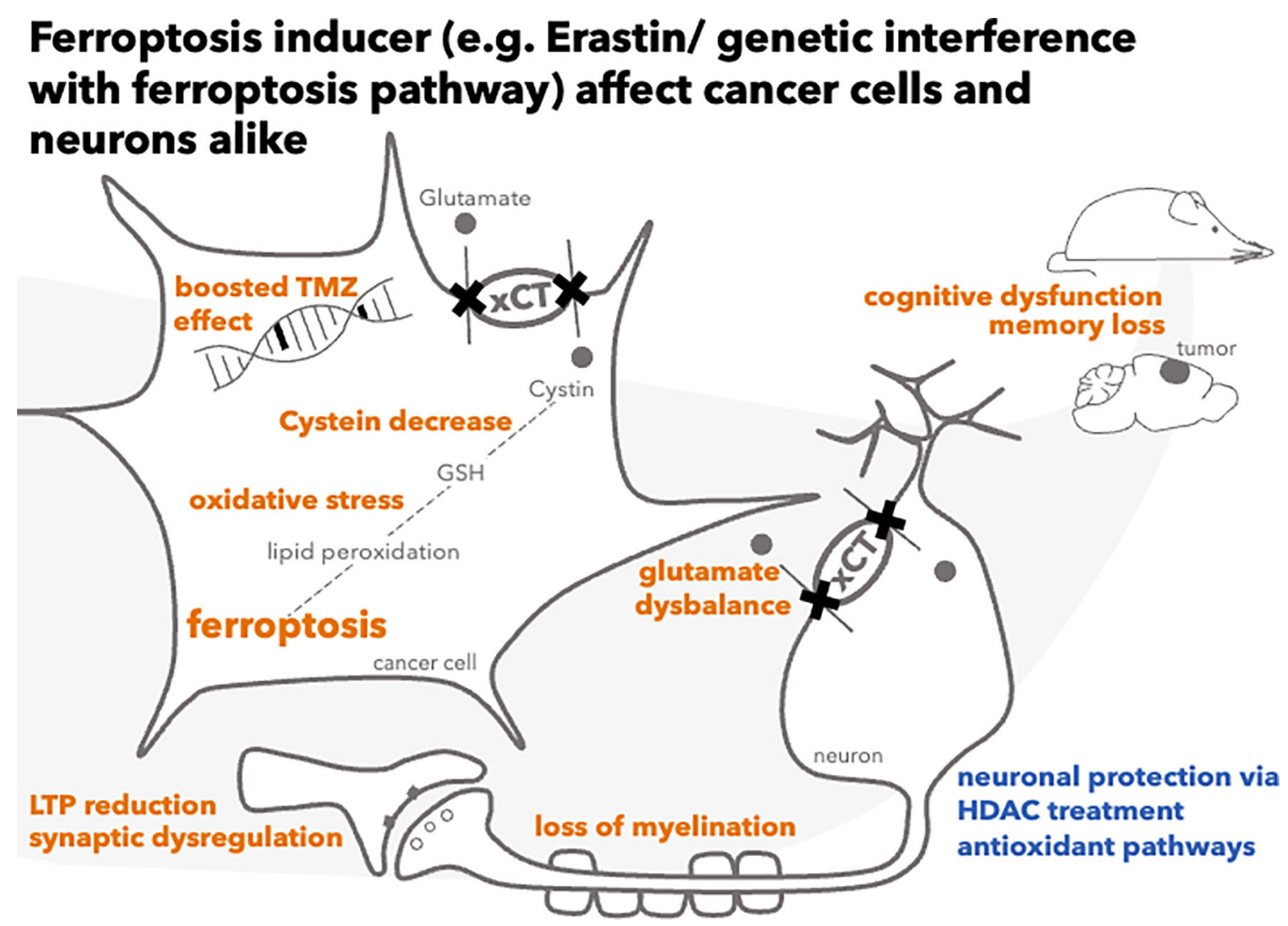

FIGURE 1 | Consequences of ferroptosis induction in cancer cells and neurons.

This bioinformatics-based immunology-ferroptosis-link was substantiated by experimental data showing that glioma GL261 cells during their early ferroptotic stages (induced by RSL3) promoted the activation of dendritic cells, which indicates a vaccination-like effect of the tumor cells on the immune system (35). With this, a link between ferroptosis and immunological responses in the context of glioma is strongly suggested and awaits further experimental clarification.

In Wan et al. the relevance of a link between ferroptosis and metabolism in the context of glioma was suggested based on a database analysis (29). For tumors, their increased metabolic reliance on utilizing amino acids (36) and lipids (37) represents malignancy hallmarks of cancer in general. In glioma, the approach of developing an amino acid-risk score - alike the here-described panels concerning ferroptosis-related genes revealed that the expression of genes involved in amino acid metabolism is important for glioma patients' survival prognosis (38). In glioma with non-mutated isocitrate dehydrogenase (IDH), branched-chain amino acids such as leucine and isoleucine, and their catabolizing enzyme branched-chain amino acid transaminase 1 (BCAT1), are more expressed - in turn, BCAT1 knock-down in glioma cells reduces the viability of glioma cells (39). Underlining the role of ferroptosis during amino acid regulation in cancer, the induction of ferroptosis eventually inhibited transcription of BCAT2 and the direct inhibition of BCAT2 led to ferroptosis in target cells (40). Additionally, cysteine and glutamate represent important amino acids during ferroptosis induction (41), whose homeostasis is interrupted by blocking xCT to achieve cell death.

In general, cancer cell growth and development are reliant on an increased lipid usage $(42,43)$. Thus, interfering with these pathways by oxidation of the lipids may boost cancer therapy by exploiting ferroptosis $(31,37)$. Increased lipid peroxidation is the result of ferroptosis induction and eventually leads to ferroptotic cell death (1). As one of the proteins that was used to generate survival-predicting ferroptosis-related genes panels (Table 1), ACSL4, increases the content of omega- 6 polyunsaturated fatty acids in cellular membranes and thus regulates how sensitive cells are to ferroptosis (44).

In one ferroptosis-signature panel, the data suggested that a risk-score built up by 19 ferroptosis genes was negatively correlated with the expression of MGMT, which confers resistance to temozolomide (26). However, many different mechanisms have been proposed to be contributing to temozolomide resistance in glioma $(5,45)$, which makes it difficult to assess their respective translational importance. Interestingly, not only coding RNA but also long non-coding RNA was shown to be predictive regarding overall survival (30). 
TABLE 1 | Risk-models using ferroptosis-related genes and their predictive capabilities.

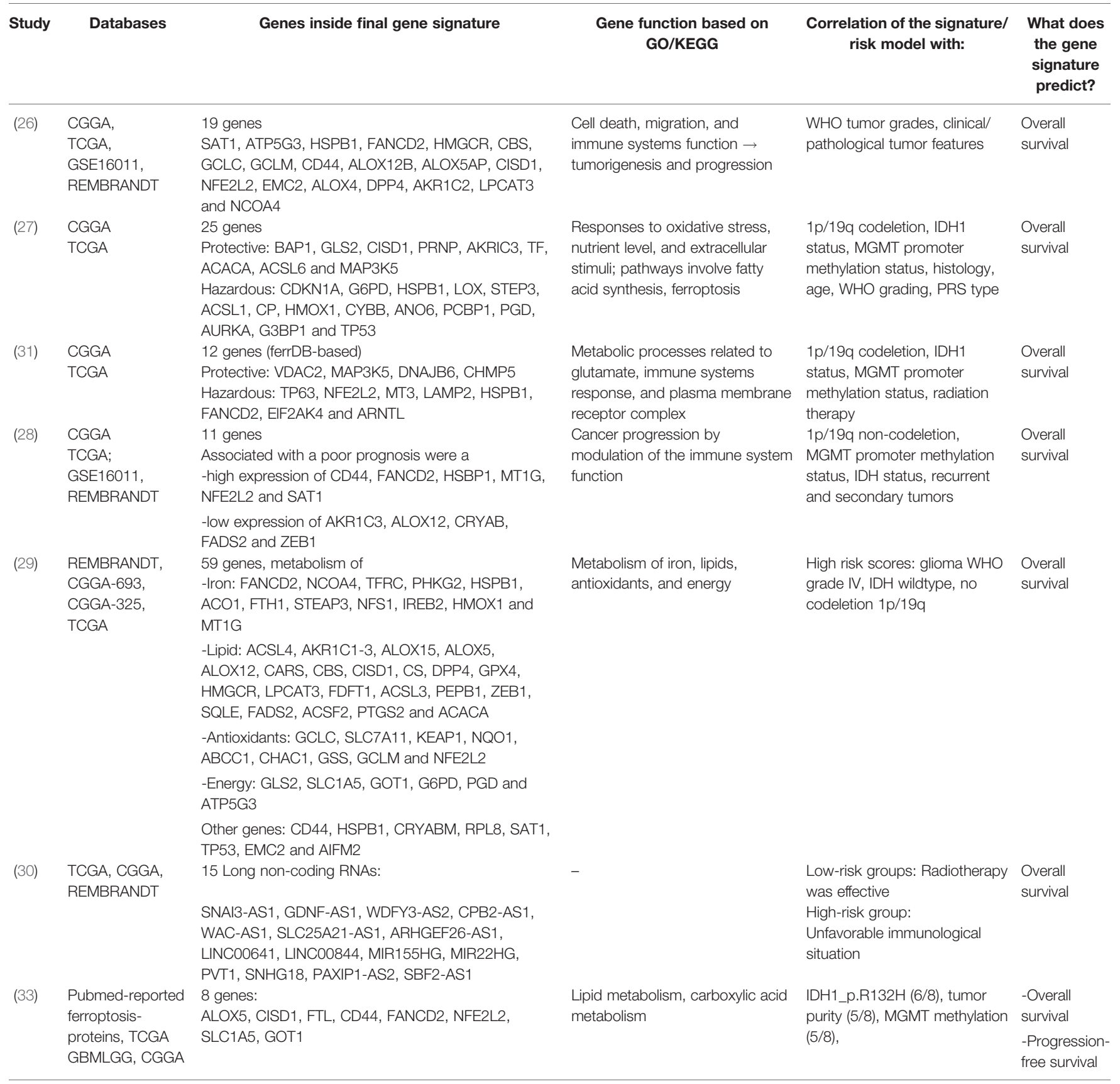

In addition to the common prediction of overall survival, one study was able to also accurately predict patients' progressionfree survival based on ferroptosis-related proteins (33).

While all presented risk models were capable of stratifying patients into high-risk and low-risk cohorts, the number of ferroptosis-related genes required to create the prognostic model substantially varies from 8 up to 59 included genes (Table 1). Redundancies of several genes between different risk models might indicate their general importance.

To evaluate if these genes are exclusively predictive of the outcome prognosis in glioma, we examined their role in comparable gene signature panels in other cancer types: A number of genes that are part of glioma risk-models (CARS, FANCD2, HMGCR, NCOA4 and SLC7A11) (46) and (AKR1C1, CARS1, CBS, CD44, CHAC1, DPP4, FANCD2, GOT1, HMGCR, SLC1A5, NCOA4 and STEAP3) (47) also accurately predicted patients' prognosis in clear renal cell carcinoma. Similarly, survival probability in hepatocellular carcinoma was reliably predicted by a glioma prediction model (ACSL3, ACSL6, ACACA, G6PD, SLC1A5, SLC7A11 and VDAC2) (48) and by a risk model with a strong overlap with the genes in the glioma models (G6PD, HMOX1, LOX, SLC7A11, STMN1/Stathmin 1) 
(49). It is, however, unlikely that ferroptosis signatures are similar across all different types of cancer, which is exemplified by a study predicting breast cancer based on a completely different set of ferroptosis-related genes (50). It will be interesting to investigate the minimum number of expressed ferroptosis-related genes in a tumor for the patients' outcome to benefit from ferroptosis induction and to investigate, how the gene expression is systematically distributed across different kinds of tumors.

\section{FERROPTOSIS IN HEALTHY NEURONS AND POTENTIAL SIDE-EFFECTS OF FERROPTOSIS INDUCTION}

The functional property of cystine/glutamate exchanger $\mathrm{xCT}$ is the uptake of cystine and the extrusion of glutamate - a key molecule of neuronal function, whose homeostasis is key for proper signal transduction and cognitive behavior $(39,51,52)$. Because another function of $\mathrm{xCT}$ is the stimulation of the antioxidative response of the cell, xCT-inhibitors can induce ferroptosis (1) (Figure 1).

Given the promising preclinical finding of improved temozolomide (Temodal ${ }^{\circledR}$, Temcad ${ }^{\circledR}$ ) chemotherapy outcome through combination with xCT-inhibitory small molecules (4), it appears necessary to also investigate such drugs' potential impact on other cells in the vicinity of the tumor tissue and in the whole body. In particular, diseases of the peripheral nervous system are known side-effects of some chemotherapeutic treatments (53), and also have been linked to ferroptosis (54).

Here, we take a closer look at the impact of xCT interference on neuronal and cognitive function (Figure 1)

An investigation of how the xCT inhibitors erastin and sorafenib affect cultured hippocampal neurons in their morphology and their vesicle pool size - a parameter tightly linked to neuronal function - has shown that such treatment could significantly disturb neuronal viability (55). In the hippocampus of xCT-deficient mice, long-term potentiation and long-term memory were impaired (56), which highlights the importance of $\mathrm{xCT}$-driven glutamate homeostasis for cognition. Although a reduction of extracellular glutamate would be expected after xCT-inhibition or deletion, additional extracellular glutamate could not reverse this effect (56). In primary hippocampal cell cultures consisting of both, neurons and glia, extracellular amino acid profiling could not confirm a reduction, but rather an increase in extracellular glutamate after erastin-induced $\mathrm{xCT}$ inhibition, suggesting a complex regulatory interplay between different cell types of the brain (55).

Inhibition of $\mathrm{xCT}$ led to a myelination defect in organotypic cerebellar slices after a few days of treatment, showing that neuronal function is disturbed also on the axonal level (57).

On a behavioral level, $\mathrm{xCT}$ was linked to stress resilience in the ventral hippocampus, because alterations in the histone acetylation status increased $\mathrm{xCT}$ expression and in turn recruited other glutamate receptors to modulate glutamate homeostasis (58). Mice with intraperitoneal erastin injections developed iron depositions in several organs such as brain, kidney and spleen, mild cerebral infarction and epithelial changes in the duodenum (59).

Efforts to examine ferroptosis-inhibitory agents to protect against such adverse effects have demonstrated that hippocampal HT22 cells could be protected from ferroptosis with Ajudecunoid C - a chemical isolated from Ajuga nipponensis via an activation of an antioxidant response element pathway (60), or with diphenylamine compounds (61). Similarly, spinal cord neurons have been protected from erastin-induced ferroptosis through LipoxinA4-induced activation of the Akt/ Nrf2/HO-1 signaling axis (62), which represents a key player in the regulating of ferroptosis and also in glioma treatment (63-65). The impact of erastin on neuronal viability was further counteracted in primary cortical neurons and SH-SY5Y cells by the iron chelator deferoxamine (66). Despite ferroptosis being similar in neurons and cancer cells, class 1 histone deacetylase inhibitors (HDACs) treatment protected neurons from ferroptosis but augmented ferroptosis in HT1080 fibrosarcoma cells (67), thereby providing the best possible outcome. This promising finding now awaits its experimental evaluation in other cell types, for example in different glioma cell lines.

Ferroptosis can also be thwarted on other levels of the ferroptosis-inducing process, for example by seleniummediated inhibition of the antioxidant glutathione peroxidase 4 (GPx4) (68), which is also implicated in the pathophysiology of glioblastoma $(7,69,70 \mathrm{~b})$. Similar to xCT-deficient mice (56), conditional deletion of GPx4 in adult forebrain neurons resulted in impaired functions of memory and spatial learning (71), and its deletion from dopaminergic midbrain neurons increased anxious behavior (72). These examples from a list of several more ferroptosis-inhibitory agents demonstrate that such drugs, initially intended to counteract neurodegeneration, could also act as support during chemotherapy to protect healthy tissue.

In contrast to erastin, which remains a purely experimental substance, multi-kinase inhibitor and ferroptosis inductor sorafenib has entered human clinical trials that included assessment of neuropsychological effects during cancer therapy. Learning, memory, and executive functions suffered over the course of treatment (73). This is further supported by a study in rats that revealed neurochemical disturbances in the hippocampus during treatment with sorafenib (74). Although the histology of the hippocampus was unaffected in that study, treatment with sorafenib for 28 days strongly decreased levels of several key metabolites such as glutamate, GABA, serine, or choline, which were measured by nuclear magnetic resonance spectroscopy. In contrast, striatum and prefrontal cortex remained rather unaffected (74). In primary rat hippocampus cultures, highperformance liquid chromatography revealed that, already after $24 \mathrm{~h}$ of sorafenib treatment, levels of glutamate, serin, and alphaaminobutyric acid were increased, and levels of glycine, cystine, and phosphoethanolamine were decreased (55). These data illustrate metabolic disturbances in response to sorafenib treatment, which may account for cognitive dysfunction.

In addition to emerging as possible side effects of ferroptosis pathway manipulation, cognitive impairment was also described as a glioblastoma symptom (75). Cognitive impairment often 
delays diagnosis and is associated with a reduced overall survival (75), which should be considered when assessing cognitive dysfunction as potential side effects of add-on drugs.

\section{CONCLUSION}

The pharmacological therapy of malignant brain tumors is difficult, especially of late-stage glioma with its treatment resistance and recurrences. The novel idea of enhancing treatment outcome through ferroptosis induction continually gains attention. Recent data uncovered a link between ferroptosis-signatures in malignant glioma and overall survival, with many studies using expression of ferroptosis-related genes to accurately predict patients' survival probability. Harnessing ferroptosis to improve tumor therapy will be an appealing approach also in malignant neuroblastoma and meningioma. But interfering with ferroptosis induction also has off-target effects, which may decrease the quality of life. Therefore, the increase in survival probability predicted by ferroptosis-gene-

\section{REFERENCES}

1. Dixon SJ, Lemberg KM, Lamprecht MR, Skouta R, Zaitsev EM, Gleason CE, et al. Ferroptosis: An Iron-Dependent Form of Nonapoptotic Cell Death. Cell (2012) 149:1060-72. doi: 10.1016/j.cell.2012.03.042

2. Cao JY, Dixon SJ. Mechanisms of Ferroptosis. Cell Mol Life Sci (2016) 73:2195-209. doi: 10.1007/s00018-016-2194-1

3. Yang WS, Stockwell BR. Ferroptosis: Death by Lipid Peroxidation. Trends Cell Biol (2016) 26:165-76. doi: 10.1016/j.tcb.2015.10.014

4. Sehm T, Rauh M, Wiendieck K, Buchfelder M, Eyupoglu IY, Savaskan NE. Temozolomide Toxicity Operates in a xCT/SLC7a11 Dependent Manner and Is Fostered by Ferroptosis. Oncotarget (2016) 7:74630-47. doi: 10.18632/ oncotarget.11858

5. Hu Z, Mi Y, Qian H, Guo N, Yan A, Zhang Y, et al. A Potential Mechanism of Temozolomide Resistance in Glioma-Ferroptosis. Front Oncol (2020) 10:897. doi: $10.3389 /$ fonc. 2020.00897

6. Weller M, Wick W, Aldape K, Brada M, Berger M, Pfister SM, et al. Glioma. Nat Rev Dis Primers (2015) 1:15017. doi: 10.1038/nrdp.2015.17

7. Yakubov E, Eibl T, Hammer A, Holtmannspötter M, Savaskan N, Steiner HH. Therapeutic Potential of Selenium in Glioblastoma. Front Neurosci (2021) 15:666679. doi: 10.3389/fnins.2021.666679

8. Liang C, Zhang X, Yang M, Dong X. Recent Progress in Ferroptosis Inducers for Cancer Therapy. Adv Mater (2019) 31:1904197. doi: 10.1002/adma.201904197

9. Huang R, Dong R, Wang N, He Y, Zhu P, Wang C, et al. Adaptive Changes Allow Targeting of Ferroptosis for Glioma Treatment. Cell Mol Neurobiol (2021). doi: 10.1007/s10571-021-01092-5. Online ahead of print

10. Zhou N, Bao J. FerrDb: A Manually Curated Resource for Regulators and Markers of Ferroptosis and Ferroptosis-Disease Associations. Database (Oxford) (2020) 2020:baaa021. doi: 10.1093/database/baaa021

11. Matthay KK, Maris JM, Schleiermacher G, Nakagawara A, Mackall CL, Diller L, et al. Neuroblastoma. Nat Rev Dis Primers (2016) 2:16078. doi: 10.1038/ nrdp. 2016.78

12. Bhoopathi P, Mannangatti P, Emdad L, Das SK, Fisher PB. The Quest to Develop an Effective Therapy for Neuroblastoma. J Cell Physiol (2021) 236:7775-91. doi: 10.1002/jcp.30384

13. Seibt TM, Proneth B, Conrad M. Role of GPX4 in Ferroptosis and Its Pharmacological Implication. Free Radic Biol Med (2019) 133:144-52. doi: 10.1016/j.freeradbiomed.2018.09.014

14. Gout PW, Buckley AR, Simms CR, Bruchovsky N. Sulfasalazine, a Potent Suppressor of Lymphoma Growth by Inhibition of the X(C)- Cystine based risk models should be traded of against potential harm through ferroptosis-inducing add-on therapy. Ideally, patients should be screened for ferroptosis-related gene expression - based on a unified set of disease-relevant ferroptosis-related genes - and stratified into high-risk or low-risk cohorts to judge their individual clinical prospects. Future clinical trials may evaluate the benefits versus side effects of ferroptosis inducing cancer treatment enhancement for different patient groups.

In summary, ferroptosis induction is a hope yielding approach to enhance antitumor therapy but requires an intricate balance between attacking the tumor and preserving the different cell types of the healthy tissue.

\section{AUTHORS CONTRIBUTIONS}

MD proposed the research. MD and JD both reviewed the literature and collected references. MD, EY, and JD wrote the manuscript and finalized the paper. All authors contributed to the article and approved the submitted manuscript.
Transporter: A New Action for an Old Drug. Leukemia (2001) 15:1633-40. doi: 10.1038/sj.leu.2402238

15. Dixon SJ, Patel DN, Welsch M, Skouta R, Lee ED, Hayano M, et al. Pharmacological Inhibition of Cystine-Glutamate Exchange Induces Endoplasmic Reticulum Stress and Ferroptosis. Elife (2014) 3:e02523. doi: 10.7554/eLife.02523

16. Hassannia B, Wiernicki B, Ingold I, Qu F, Van Herck S, Tyurina YY, et al. Nano-Targeted Induction of Dual Ferroptotic Mechanisms Eradicates HighRisk Neuroblastoma. J Clin Invest. (2018) 128:3341-55. doi: 10.1172/JCI99032

17. De Jong WH, Borm PJ. Drug Delivery and Nanoparticles: Applications and Hazards. Int J Nanomed (2008) 3:133-49. doi: 10.2147/IJN.S596

18. Geng N, Shi BJ, Li SL, Zhong ZY, Li YC, Xua WL, et al. Knockdown of Ferroportin Accelerates Erastin-Induced Ferroptosis in Neuroblastoma Cells. Eur Rev Med Pharmacol Sci (2018) 22:3826-36. doi: 10.26355/eurrev_201806_15267

19. Floros KV, Cai J, Jacob S, Kurupi R, Fairchild CK, Shende M, et al. MYCNAmplified Neuroblastoma Is Addicted to Iron and Vulnerable to Inhibition of the System Xc-/Glutathione Axis. Cancer Res (2021) 81:1896-908. doi: 10.1158/0008-5472.CAN-20-1641

20. Lu Y, Yang Q, Su Y, Ji Y, Li G, Yang X, et al. MYCN Mediates TFRCDependent Ferroptosis and Reveals Vulnerabilities in Neuroblastoma. Cell Death Dis (2021) 12:511. doi: 10.1038/s41419-021-03790-w

21. Monteleone L, Speciale A, Valenti GE, Traverso N, Ravera S, Garbarino O, et al. Pkco Inhibition as a Strategy to Sensitize Neuroblastoma Stem Cells to Etoposide by Stimulating Ferroptosis. Antioxidants (2021) 10:691. doi: 10.3390/antiox10050691

22. Lu R, Jiang Y, Lai X, Liu S, Sun L, Zhou ZW. A Shortage of FTH Induces ROS and Sensitizes RAS-Proficient Neuroblastoma N2A Cells to Ferroptosis. Int J Mol Sci (2021) 22:8898. doi: 10.3390/ijms22168898

23. Bao Z, Hua L, Ye Y, Wang D, Li C, Xie Q, et al. MEF2C Silencing Downregulates NF2 and E-Cadherin and Enhances Erastin-Induced Ferroptosis in Meningioma. Neuro Oncol (2021) noab114. doi: 10.1093/ neuonc/noab114

24. Marosi C. Will Mastering Ferroptosis Allow Treating Refractory Meningiomas? Neuro Oncol (2021). doi: 10.1093/neuonc/noab218

25. Noch EK, Ramakrishna R, Magge R. Challenges in the Treatment of Glioblastoma: Multisystem Mechanisms of Therapeutic Resistance. World Neurosurg (2018) 116:505-17. doi: 10.1016/j.wneu.2018.04.022

26. Liu HJ, Hu HM, Li GZ, Zhang Y, Wu F, Liu X, et al. Ferroptosis-Related Gene Signature Predicts Glioma Cell Death and Glioma Patient Progression. Front Cell Dev Biol (2020) 8:538. doi: 10.3389/fcell.2020.00538 
27. Zhuo S, Chen Z, Yang Y, Zhang J, Tang J, Yang K. Clinical and Biological Significances of a Ferroptosis-Related Gene Signature in Glioma. Front Oncol (2020) 10:590861. doi: 10.3389/fonc.2020.590861

28. Chen Z, Wu T, Yan Z, Zhang M. Identification and Validation of an 11Ferroptosis Related Gene Signature and Its Correlation With Immune Checkpoint Molecules in Glioma. Front Cell Dev Biol (2021) 9:652599. doi: 10.3389/fcell.2021.652599

29. Wan RJ, Peng W, Xia QX, Zhou HH, Mao XY. Ferroptosis-Related Gene Signature Predicts Prognosis and Immunotherapy in Glioma. CNS Neurosci Ther (2021) 27:973-86. doi: 10.1111/cns.13654

30. Zheng J, Zhou Z, Qiu Y, Wang M, Yu H, Wu Z, et al. A Prognostic Ferroptosis-Related IncRNAs Signature Associated With Immune Landscape and Radiotherapy Response in Glioma. Front Cell Dev Biol (2021) 9:675555. doi: 10.3389/fcell.2021.675555

31. Zheng Y, Ji Q, Xie L, Wang C, Yu CN, Wang YL, et al. Ferroptosis-Related Gene Signature as a Prognostic Marker for Lower-Grade Gliomas. J Cell Mol Med (2021) 25:3080-90. doi: $10.1111 / \mathrm{jcmm} .16368$

32. Zhu X, Zhou Y, Ou Y, Cheng Z, Han D, Chu Z, et al. Characterization of Ferroptosis Signature to Evaluate the Predict Prognosis and Immunotherapy in Glioblastoma. Aging (2021) 13:17655-72. doi: 10.18632/aging.203257

33. Liu Y, Xu Z, Jin T, Xu K, Liu M, Xu H. Ferroptosis in Low-Grade Glioma: A New Marker for Diagnosis and Prognosis. Med Sci Monit (2020) 26:e921947. doi: 10.12659/MSM.921947

34. Deng S, Zheng Y, Mo Y, Xu X, Li Y, Zhang Y, et al. Ferroptosis Suppressive Genes Correlate With Immunosuppression in Glioblastoma. World Neurosurg (2021) 152:e436-48. doi: 10.1016/j.wneu.2021.05.098

35. Efimova I, Catanzaro E, van der Meeren L, Turubanova VD, Hammad H, Mishchenko TA, et al. Vaccination With Early Ferroptotic Cancer Cells Induces Efficient Antitumor Immunity. J Immunother Cancer (2020) 8: e001369. doi: 10.1136/jitc-2020-001369

36. Wei Z, Liu X, Cheng C, Yu W, Yi P. Metabolism of Amino Acids in Cancer. Front Cell Dev Biol (2021) 8:603837. doi: 10.3389/fcell.2020.603837

37. Cheng C, Geng F, Cheng X, Guo D. Lipid Metabolism Reprogramming and its Potential Targets in Cancer. Cancer Commun (Lond) (2018) 38:27. doi: 10.1186/s40880-018-0301-4

38. Liu YQ, Chai RC, Wang YZ, Wang Z, Liu X, Wu F, et al. Amino Acid Metabolism-Related Gene Expression-Based Risk Signature can Better Predict Overall Survival for Glioma. Cancer Sci (2019) 110:321-33. doi: 10.1111/ cas. 13878

39. Koppula P, Zhuang L, Gan B. Cystine Transporter SLC7A11/xCT in Cancer: Ferroptosis, Nutrient Dependency, and Cancer Therapy. Protein Cell (2021) 12:599-620. doi: 10.1007/s13238-020-00789-5

40. Wang K, Zhang Z, Tsai HI, Liu Y, Gao J, Wang M, et al. Branched-Chain Amino Acid Aminotransferase 2 Regulates Ferroptotic Cell Death in Cancer Cells. Cell Death Differ (2021) 28:1222-36. doi: 10.1038/s41418-020-00644-4

41. Daher B, Vučetić M, Pouysségur J. Cysteine Depletion, a Key Action to Challenge Cancer Cells to Ferroptotic Cell Death. Front Oncol (2020) 10:723. doi: $10.3389 /$ fonc. 2020.00723

42. Strickland M, Stoll EA. Metabolic Reprogramming in Glioma. Front Cell Dev Biol (2017) 5:43. doi: 10.3389/fcell.2017.00043

43. Li D, Li Y. The Interaction Between Ferroptosis and Lipid Metabolism in Cancer. Signal Transduct Targeted Ther (2020) 5:108. doi: 10.1038/s41392-020-00216-5

44. Doll S, Proneth B, Tyurina YY, Panzilius E, Kobayashi S, Ingold I, et al. ACSL4 Dictates Ferroptosis Sensitivity by Shaping Cellular Lipid Composition. Nat Chem Biol (2017) 13:91-8. doi: 10.1038/nchembio.2239

45. Singh N, Miner A, Hennis L, Mittal S. Mechanisms of Temozolomide Resistance in Glioblastoma - A Comprehensive Review. Cancer Drug Resist (2021) 4:17-43. doi: 10.20517/cdr.2020.79

46. Wu G, Wang Q, Xu Y, Li Q, Cheng L. A New Survival Model Based on Ferroptosis-Related Genes for Prognostic Prediction in Clear Cell Renal Cell Carcinoma. Aging (2020) 12:14933-48. doi: 10.18632/aging.103553

47. Hong Y, Lin M, Ou D, Huang Z, Shen P. A Novel Ferroptosis-Related 12Gene Signature Predicts Clinical Prognosis and Reveals Immune Relevancy in Clear Cell Renal Cell Carcinoma. BMC Cancer (2021) 21:831. doi: 10.1186/ s12885-021-08559-0

48. Zhang H, Liu R, Sun L, Guo W, Hu X. The Effect of Ferroptosis-Related Genes on Prognosis and Tumor Mutational Burden in Hepatocellular Carcinoma. J Oncol (2021) 2021:7391560. doi: 10.1155/2021/7391560
49. Wan S, Lei Y, Li M, Wu B. A Prognostic Model for Hepatocellular Carcinoma Patients Based on Signature Ferroptosis-Related Genes. Hepatol Int (2021) doi. doi: 10.1007/s12072-021-10248-w. Online ahead of print

50. Zhu L, Tian Q, Jiang S, Gao H, Yu S, Zhou Y, et al. A Novel FerroptosisRelated Gene Signature for Overall Survival Prediction in Patients With Breast Cancer. Front Cell Dev Biol (2021) 9:670814. doi: 10.3389/fcell.2021.670184

51. Bridges R, Lutgen V, Lobner D, Baker DA. Thinking Outside the Cleft to Understand Synaptic Activity: Contribution of the Cystine-Glutamate Antiporter (System Xc-) to Normal and Pathological Glutamatergic Signaling. Pharmacol Rev (2012) 64:780-802. doi: 10.1124/pr.110.003889

52. Diering GH, Huganir RL. The AMPA Receptor Code of Synaptic Plasticity. Neuron (2018) 100:314-29. doi: 10.1016/j.neuron.2018.10.018

53. Li T, Mizrahi D, Goldstein D, Kiernan MC, Park SB. Chemotherapy and Peripheral Neuropathy. Neurol Sci (2021) 42:4109-21. doi: 10.1007/s10072021-05576-6

54. Wang H, Huo X, Han C, Ning J, Chen H, Li B, et al. Ferroptosis Is Involved in the Development of Neuropathic Pain and Allodynia. Mol Cell Biochem (2021) 476:3149-61. doi: 10.1007/s11010-021-04138-w

55. Dahlmanns M, Yakubov E, Chen D, Sehm T, Rauh M, Savaskan N, et al. Chemotherapeutic xCT Inhibitors Sorafenib and Erastin Unraveled With the Synaptic Optogenetic Function Analysis Tool. Cell Death Discovery (2017) 3:17030. doi: 10.1038/cddiscovery.2017.30

56. Li Y, Tan Z, Li Z, Sun Z, Duan S, Li W. Impaired Long-Term Potentiation and Long-Term Memory Deficits in xCT-Deficient Sut Mice. Biosci Rep (2012) 32:315-21. doi: 10.1042/BSR20110107

57. Soria FN, Zabala A, Pampliega O, Palomino A, Miguelez C, Ugedo L, et al. Cystine/glutamate Antiporter Blockage Induces Myelin Degeneration. Glia (2016) 64:1381-95. doi: 10.1002/glia.23011

58. Nasca C, Bigio B, Zelli D, De Angelis P, Lau T, Okamoto M, et al. Role of the Astroglial Glutamate Exchanger xCT in Ventral Hippocampus in Resilience to Stress. Neuron (2017) 96:402-13.e405. doi: 10.1016/j.neuron.2017.09.020

59. Zhao J, Xu B, Xiong Q, Feng Y, Du H. Erastin-induced Ferroptosis Causes Physiological and Pathological Changes in Healthy Tissues of Mice. Mol Med Rep (2021) 24:713. doi: 10.3892/mmr.2021.12352

60. Tan Q, Fang Y, Peng X, Zhou H, Xu J, Gu Q. A New Ferroptosis Inhibitor, Isolated From Ajuga Nipponensis, Protects Neuronal Cells via Activating NRF2-Antioxidant Response Elements (AREs) Pathway. Bioorg Chem (2021) 115:105177. doi: 10.1016/j.bioorg.2021.105177

61. Hinder L, Pfaff AL, Emmerich RE, Michels S, Schlitzer M, Culmsee C. Characterization of Novel Diphenylamine Compounds as Ferroptosis Inhibitors. J Pharmacol Exp Ther (2021) 378:184-96. doi: 10.1124/jpet.121.000534

62. Wei N, Lu T, Yang L, Dong Y, Liu X. Lipoxin A4 Protects Primary Spinal Cord Neurons From Erastin-Induced Ferroptosis by Activating the Akt/Nrf2/HO-1 Signaling Pathway. FEBS Open Bio (2021) 11:2118-26. doi: 10.1002/22115463.13203

63. Fan Z, Wirth AK, Chen D, Wruck CJ, Rauh M, Buchfelder M, et al. Nrf2Keap1 Pathway Promotes Cell Proliferation and Diminishes Ferroptosis. Oncogenesis (2017) 6:e371. doi: 10.1038/oncsis.2017.65

64. Villalpando-Rodriguez GE, Blankstein AR, Konzelman C, Gibson SB. Lysosomal Destabilizing Drug Siramesine and the Dual Tyrosine Kinase Inhibitor Lapatinib Induce a Synergistic Ferroptosis Through Reduced Heme Oxygenase-1 (HO-1) Levels. Oxid Med Cell Longev (2019) 2019:9561281. doi: 10.1155/2019/9561281

65. Gao X, Guo N, Xu H, Pan T, Lei H, Yan A, et al. Ibuprofen Induces Ferroptosis of Glioblastoma Cells via Downregulation of Nuclear Factor Erythroid 2Related Factor 2 Signaling Pathway. Anticancer Drugs (2020) 31:27-34. doi: 10.1097/CAD.0000000000000825

66. Zhang Y, Fan BY, Pang YL, Shen WY, Wang X, Zhao CX, et al. Neuroprotective Effect of Deferoxamine on Erastininduced Ferroptosis in Primary Cortical Neurons. Neural Regener Res (2020) 15:1539-45. doi: 10.4103/1673-5374.274344

67. Zille M, Kumar A, Kundu N, Bourassa MW, Wong VSC, Willis D, et al. Ferroptosis in Neurons and Cancer Cells Is Similar But Differentially Regulated by Histone Deacetylase Inhibitors. eNeuro (2019) 6: ENURO.0263-18.2019. doi: 10.1523/ENEURO.0263-18.2019

68. Alim I, Caulfield JT, Chen Y, Swarup V, Geschwind DH, Ivanova E, et al. Selenium Drives a Transcriptional Adaptive Program to Block Ferroptosis and Treat Stroke. Cell (2019) 177:1262-79.e1225. doi: 10.1016/j.cell.2019.03.032 
69. Yakubov E, Buchfelder M, Eyüpoglu IY, Savaskan NE. Selenium Action in Neuro-Oncology. Biol Trace Elem Res (2014) 161:246-54. doi: 10.1007/s12011014-0111-8

70. Yakubov E. Der Einfluss Des Zerebralen Selenspiegels Auf Die Progression Maligner Hirntumoren. Dissertation. Erlangen: Friedrich-Alexander University of Erlangen-Nürnberg (2019).

71. Hambright WS, Fonseca RS, Chen L, Na R, Ran Q. Ablation of Ferroptosis Regulator Glutathione Peroxidase 4 in Forebrain Neurons Promotes Cognitive Impairment and Neurodegeneration. Redox Biol (2017) 12:8-17. doi: 10.1016/j.redox.2017.01.021

72. Schriever SC, Zimprich A, Pfuhlmann K, Baumann P, Giesert F, Klaus V, et al. Alterations in Neuronal Control of Body Weight and Anxiety Behavior by Glutathione Peroxidase 4 Deficiency. Neuroscience (2017) 357:241-54. doi: 10.1016/j.neuroscience.2017.05.050

73. Mulder SF, Bertens D, Desar IM, Vissers KC, Mulders PF, Punt CJ, et al. Impairment of Cognitive Functioning During Sunitinib or Sorafenib Treatment in Cancer Patients: A Cross Sectional Study. BMC Cancer (2014) 14:219. doi: 10.1186/1471-2407-14-219

74. Du C, Shao X, Zhu R, Li Y, Zhao Q, Fu D, et al. NMR-Based Metabolic Profiling Reveals Neurochemical Alterations in the Brain of Rats Treated With Sorafenib. Neurotox Res (2015) 28:290-301. doi: 10.1007/s12640-015-9539-7
75. Bruhn H, Blystad I, Milos P, Malmström A, Dahle C, Vrethem M, et al. Initial Cognitive Impairment Predicts Shorter Survival of Patients With Glioblastoma. Acta Neurol Scand (2021). doi: 10.1111/ane.13529

Conflict of Interest: The authors declare that the research was conducted in the absence of any commercial or financial relationships that could be construed as a potential conflict of interest.

Publisher's Note: All claims expressed in this article are solely those of the authors and do not necessarily represent those of their affiliated organizations, or those of the publisher, the editors and the reviewers. Any product that may be evaluated in this article, or claim that may be made by its manufacturer, is not guaranteed or endorsed by the publisher.

Copyright (c) 2021 Dahlmanns, Yakubov and Dahlmanns. This is an open-access article distributed under the terms of the Creative Commons Attribution License (CC BY). The use, distribution or reproduction in other forums is permitted, provided the original author(s) and the copyright owner(s) are credited and that the original publication in this journal is cited, in accordance with accepted academic practice. No use, distribution or reproduction is permitted which does not comply with these terms. 


\section{GLOSSARY}

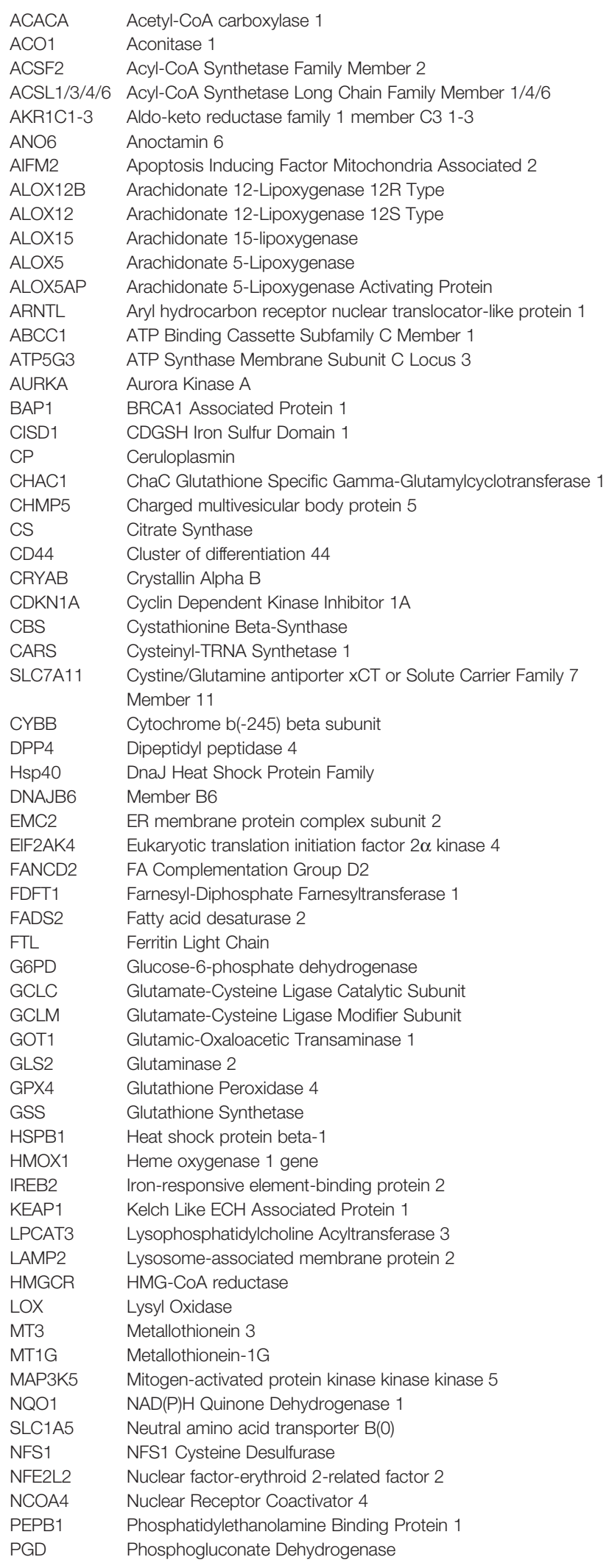

(Continued)
Continued

PHKG2 Phosphorylase Kinase Catalytic Subunit Gamma 2

PRNP Prion protein

PTGS2 Prostaglandin-Endoperoxide Synthase 2

G3BP1 Ras GTPase-activating protein-binding protein 1

RB1 RB Transcriptional Corepressor 1

RPL8 Ribosomal Protein L8

STEAP3 Six-transmembrane epithelial antigen of the prostate 3

SAT1 Spermidine/Spermine N1-Acetyltransferase 1

SQLE Squalene Epoxidase

TFRC Transferrin Receptor

TP53 Tumor protein p53

TP63 Tumor protein p63

VDAC2 Voltage-dependent anion-selective channel protein 2

ZEB1 Zinc Finger E-Box Binding Homeobox 1 